\title{
Participación de la Dra. Alejandrina Mata Segreda, Decana de la Facultad de Educación
}

\author{
UNIVERSIDAD DE COSTA RICA \\ FACULTAD DE EDUCACIÓN
}

Celebración del 40 Aniversario de la Revista Educación

El primer número de la Revista Educación de la Universidad de Costa Rica se publicó en el año 1977, con la dirección de la Licda. Flor de María Pérez Zamora y el Consejo Editorial integrado por las profesoras y los profesores María Eugenia Dengo de Vargas, Isaac Felipe Azofeifa, Jesús Ugalde, Yolanda Rojas y Carlos Moya. Si bien la revista contó con el sello editorial de la Editorial Universidad de Costa Rica, su diagramación, diseño, levantado de textos, corrección de pruebas, impresión y encuadernación se realizó en los talleres gráficos de la Editorial Fernández Arce, con la responsabilidad del profesor Mario Fernández Lobo. Don Mario escribió la presentación de la revista destacando la necesidad de contar con un órgano especializado para la difusión de conocimiento en materia educativa y expone los objetivos que orientarían esta publicación, ocho en total, referidos a la difusión del conocimiento generado por medio de la investigación y del análisis crítico de posiciones teóricas de actualidad, favorecer el mejoramiento didáctico y la actitud vigilante de profesionales en educación en lo que respecta al cambio, interpretar el contexto educativo nacional y colaborar con la política de proyección social de la Universidad de Costa Rica.

Este número constituyó un homenaje a la Dra. Emma Gamboa, quien había fallecido un año antes; pero había dejado preparado un artículo para la revista sobre libros de texto básicos para la enseñanza de la lectura, según se interpreta de la dedicatoria que elaboró su directora, la profesora Flor de María Pérez. Doña María Eugenia Dengo se refirió a la personalidad de Emma Gamboa. Se publicó también la oración fúnebre que don Claudio Gutiérrez, rector de la Universidad en el momento, había compartido en la despedida. Como a doña Emma le gustaba hablar "en modo poético", se incluyeron dos de sus poemas, uno referido a la realidad de un sueño: la construcción del edificio de la Facultad de Educación. El otro a su otro sueño: la creación de la Escuela Nueva, que hoy lleva el nombre de Escuela Nueva Laboratorio Emma Gamboa.

A partir de allí, la revista incluye varios artículos que reflejan las tendencias teóricas que en esa década se "cocinaban" en la Facultad de Educación, pues, además del mencionado artículo de doña Emma, el profesor Mario Fernández habla sobre la moderna enseñanza de la lengua y los libros de texto de español, la profesora Elsa Orozco expone sobre la enseñanza de los idiomas extranjeros, el profesor Isaac Felipe Azofeifa polemiza sobre la educación cívica, el Dr. Phillip P. Johnson, junto con los profesores Juan Manuel Esquivel y Hugo Mora Poltronieri, se refieren a la enseñanza individualizada, y la profesora Victoria Garrón de Doryan trata el tema de la educación permanente como uno de los principales "caballos de batalla" de la Unesco. La revista cerró con el artículo de una joven profesora, Sonia Carballo de Hernández, quien presentó el tema de la enseñanza centrada en el alumnado. 
Tres artículos más deseo comentar por aparte, pues, a mi juicio, constituyen una especie de anomalía académica de la época, no solo por la edad que en ese momento tenían los dos profesores y la profesora responsables de ellos, sino porque presentaban temas poco estudiados o porque rompían barreras geográficas. Con respecto a esto último, la profesora Yolanda Rojas reflexionaba, nada más que eso, sobre la formación de un educador o educadora para Centroamérica. El profesor Jesús Ugalde, con los pies puestos en el hoy de aquel entonces, analizaba las implicaciones para el futuro educativo. Y el profesor Carlos German Paniagua ampliaba su mirada hacia la universidad latinoamericana desde la perspectiva de la didáctica universitaria.

Este primer número inicia la tradición gráfica de la revista, con esa pésima fotografía de la escultura del maestro Mauro Fernández inserta en un diseño rígido y cuadrado que, con escasas modificaciones, se mantuvo durante dos décadas. La dirección de la revista inicialmente la tenía Flor de María y luego don Jesús; el Consejo Editorial sufrió pocas modificaciones (en algún momento se incorporaron la profesora Ana Cecilia Hernández y el profesor Juan Manuel Esquivel), hasta el año 1982 en que cambió radicalmente. En ese momento se conforma con la participación de la profesora Nora Ramírez de Chacón y los profesores Albam Brenes y Gerardo Fonseca, recién graduado en el exterior, quedando de la vieja guardia, solamente, Juan Manuel Esquivel. Y aparece un personaje externo, don Rafael Ángel Herra.

En la década de los años noventa, la dirección de la revista la asume la profesora Nidia García Lizano y al Consejo Editorial se integran nuevos profesores y nuevas profesoras, principalmente aquellas personas que regresaban a la Universidad después de obtener sus títulos de posgrado en el exterior, como las profesoras Ileana Contreras y Ana Cecilia Torres y el profesor Walter Salazar. Al pensionarse la profesora García, recuerdo que llegó a la oficina del Decanato de la Facultad a solicitar dos cosas, la primera la necesidad de buscar a una persona que asumiera la dirección de la revista con un Consejo Editorial renovado; la segunda, llamar la atención de que las instalaciones físicas de la revista se encontraban en su propio escritorio y, ahora que se pensionaba, ¿dónde iban a estar?

Así llegó el año 1997 y nuestra revista cambió de cara. Recuerdo que la profesora Marta Rojas, cuando fue a presentarse al Decanato como nueva directora de la revista, en ese momento me dijo que su primera acción sería modificar la portada. Aunque reconocía el valor de don Mauro como reformador de la educación costarricense, la revista no podía seguir presentando esa pésima fotografía suya, además, los tiempos habían cambiado. Y cambió todo, el Consejo Editorial con gente totalmente nueva y más joven tendiendo hacia la representación de cada una de las unidades académicas de la Facultad de Educación, incluido ya el IIMEC que asumió la responsabilidad de su gestión y albergue físico con la participación de Marta; se incorpora la figura de la editora de la revista que fue la señora María Elena Camacho. A partir del 2004, ya se incorporan al Consejo Editorial académicos totalmente externos al país y a la universidad, Juan Carlos Tedesco de la Unesco y Oscar Soriano de la Universidad Pedagógica Francisco Morazán de Honduras.

En cuanto a la imagen, la revista se vistió de un color suave, entre celeste y gris, incorporando grabados, pintura, escultura, fotografía; en fin, cada número conservaba su imagen corporativa, sin embargo, con un elemento central diferente en cada uno de ellos. En el año 2009 asume la dirección de la revista la profesora Flora Salas y en el 2010 la profesora Carmen Grace Salazar. 
La era de las tecnologías digitales no ha dejado a nadie quieto, por lo que igualmente tocó, molestó y modificó a nuestra Revista Educación. En la segunda década del Siglo XXI la revista cambia de cara nuevamente con un diseño y un logo diferentes, y con su flamante dirección electrónica en la portada se involucra generando la digitalización de todos sus números bajo el liderazgo de su actual directora, la profesora Lastenia Bonilla. Hoy en día, cualquiera de ellos puede ser consultado ingresando al sitio del Portal de las Revistas Académicas de la Universidad de Costa Rica. Podrán corroborar que allí pueden encontrar desde los poemas de doña Emma hasta el último número de este año. Pero no nos quedamos allí, la revista se volvió ambientalmente responsable e internacionalmente accesible cuando a partir del año 2013 solo se edita en formato digital. Si bien, a quienes nos gusta tener un libro de papel en las manos este cambio nos afecta, la gran mayoría de docentes y estudiantes, y de la comunidad académica mundial se siente mucho más cómoda, porque ahora no deberán pagar ni los setenta y cinco, ni los mil o los dos mil colones que terminó costando cada número, ni tendrán que desplazarse a una biblioteca física para consultar los números de la revista. Durante este siglo, la dinámica de renovación del Consejo Editorial se ha mantenido, muchos hemos asumido esa responsabilidad de orientar el rumbo de nuestra revista.

Y sobre este rumbo académico destaco grandes características:

1. La revista sirvió de nicho inicialmente para que el personal académico de la Facultad de Educación diera a conocer su trabajo. En sus primeros números se percibe un aire fundamentalmente de experiencia docente que se sistematiza con el apoyo de insumos teóricos de avanzada.

2. La tendencia positivista de la investigación en educación se observa al pasar las páginas de los números de la revista durante, al menos, dos décadas, en las que se observan tablas y gráficos, fórmulas estadísticas para la definición de muestras y conclusiones fundamentadas en el conocimiento de lo real y objetivo.

3. Lo cualitativo viene a irrumpir más adelante con largos textos interpretativos y temas que trascienden lo que antes se había considerado exclusivamente como tema educativo. Es decir, el fenómeno educativo se sale de las aulas escolares y se observa, analiza e investiga en otros espacios fuera de la escuela. No solo se observa una apertura teórica, sino que la revista va dibujando, página a página, la amplitud de temas alrededor de los cuales esta Facultad forma educadores y educadoras.

4. Nuestra revista ya no es solo nuestra. Se ha abierto al mundo académico, y porque atrae o porque nos hemos comprometido con estándares de calidad internacional, por lo menos la mitad de los artículos que se publican hoy día evidencia autores y autoras del extranjero.

5. Algunos de los descriptores de los artículos de la revista de los últimos años reflejan la diversidad de temas que se publican, destaco algunos de ellos: género, actividad física, gasto energético, terrorismo, educación secundaria, libros de texto, liderazgo, gestión del conocimiento, organizaciones inteligentes, bilingüismo, comunicación, ideología, teoría de la actividad, teoría de sistemas, primates, tropa, integración/ inclusión, educación a distancia, internacionalización de la educación, resistencias, comunidades de aprendizaje, investigación - acción, formación del profesorado, recreación, alfabetización tecnológica, tecnología móvil, educación musical, desarrollo 
humanos, estrategia didáctica, salud pública, derecho a la educación, hidrología subterránea, modelo de negocio, módulo didáctico, recreo escolar, violencia entre pares, modelos de universidades, pensamiento crítico...

¿Cómo terminar esta exposición? Sin terminarla, dejando abierto el camino para que la historia de la Revista Educación se continúe contando, que su identidad, ahora global, promueva el intercambio de conocimiento y experiencias en educación a partir de las expectativas internacionales de lo que es una buena revista académica, a la vez que refleje la identidad del desarrollo teórico de la educación en la Universidad de Costa Rica y en los espacios académicos de quienes publican en ella. 